\title{
EDITORIAL
}

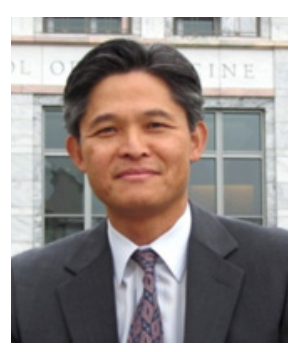

\section{Special Issue on Mechanobiology and Diseases}

\author{
Hanjoong Jo
}

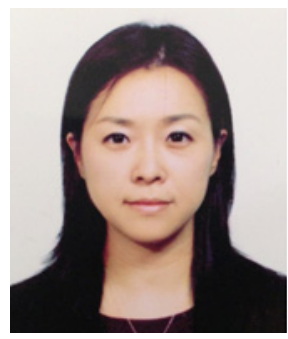

Jennifer H. Shin

(C) The Korean Society of Medical \& Biological Engineering and Springer 2015

Most cells in our body sense and respond to mechanical stimuli to regulate their physiological processes. These mechanical forces can be imposed externally by the environmental factors or internally by natural microenvironment of cells' niche. Direct forces include shear stress on endothelial cells from blood flow, cyclic tensile stress on heart muscle cells from beating heart, and compressive loading on chondrocytes from one's own weight. Indirect forces include natural tissue alignment in the muscles and ligaments as well as the stiffness of extracellular microenvironment that varies depending on tissue types. These mechanical forces regulate the physiological functions of the cells that are under immediate influence. And generally normal cells in our body possess their own response strategies against moderate physicochemical stresses for tissue homeostasis. When the stress level falls below or goes above a critical threshold, pathological responses may be initiated to prompt disease conditions.

Gravity is one of the major mechanical forces our body is

Hanjoong Jo

Department of Biomedical Engineering at Georgia Institute of Technology and Emory University, USA

Tel : +1-404-712-9654 / Fax : +1-404-727-3330

E-mail : hjo@bme.gatech.edu

Jennifer H. Shin $(\bowtie)$

Department of Mechanical Engineering, KAIST, Korea

Tel : +82-42-350-3232 / Fax : +82-42-350-3210

E-mail :j_shin@kaist.ac.kr naturally adapted to. For example, bone tissues are known to adapt their structure, leading to alterations in the density and stiffness in accordance to compressive or tensile loading due to gravitational force or movements. Such adaptation of bone tissue involves alterations in physiological functions of the bone cells. Upon a prolonged bed rest, patients experience significant loss in their bone mass, especially at the heel bone and lower limb. These cells are used to having a certain level of loading from the body weight, and thus sudden removal of such natural force would result in degeneration. Similarly, lack of physical activities can lead to significant muscle loss, developing a medical condition called muscle atrophy. Astronauts can develop decreased muscle tone after just a few days of weightlessness. In addition to stresses originated from the gravitational field, most of the cells in our body experience shear stress from bodily fluids at various levels. In particular, the endothelial cells on the vascular wall and heart valves are constantly subjected to shear stress, which regulates important vascular physiology. Abnormalities in flow conditions can lead to development of atherosclerosis and heart valve disease in specific regions of low shear where white cells adhere and accumulate. Most of the connective tissues consist of water-filled matrices, and thus the interstitial flow within the matrices acts as critical regulators of resident cells such as vascular muscle cells, valve interstitial cells, chondrocytes, osteocytes, or fibroblasts. Tensile force is another prevalent stress imposed on cells, 
especially those of the epithelial type. Skin, for example, is the largest organ in our body that expands to cover the growing skeleton and soft tissue. Especially in the case of wound, the skin must migrate to cover the denuded area. Thus, cells in the epidermal skin are constantly subjected to intrinsic tensile stresses from growth and healing as well as external mechanical tensile, compressive, or shearing stresses from body movement and contacts.

Despite the importance of mechanical stresses in cellular physiology and pathology, the current focus of medicine largely ignores the physical basis of diseases. Mechanobiology is an interdisciplinary field that combines conventional mechanical perspectives with various fields of biology. At the center of mechanobiology, there resides a concept called mechanosensing and mechanotransduction, which is the way cells sense and respond to mechanical stimuli. Proper mechanotransduction starts from sensing of changes in mechanical forces imposed on cells by mechano-receptors, which reside in various cellular regions (luminal receptors such as ion channels, G-protein coupled receptors, glycocalyx, caveolae, primary cilia; cell-cell junctional receptors such as PECAM-1 and VE-Cadherin; abluminal receptors for extracellular matrix integrins; and intracellular actincytoskeletons). Once detected by various mechanosensors, the mechanical force signal is transmitted through a complex intracellular signaling cascade leading to diverse cellular responses including gene expression changes in the nucleus. Main goal of the mechanobiology is to understand how mechanical forces regulate not only normal cell physiology but also pathobiology of diseases so it may be utilized in developing diagnostics or therapeutics.

This special issue "Mechanobiology and Disease" tries to capture various scopes of mechanobiology and diseases, with a special emphasis on the most representative mechanobiological disease, atherosclerosis.

For decades, animal models have been used to allow better understanding of the human diseases. As one example, animal models of atherosclerosis are important tools to understand the pathophysiology of atherosclerosis and identify new therapies. Various types of animal models have been used throughout the years, including different species such as pigeon, mice, rabbit, pig and nonhuman primates. Each model may be used to elucidate a specific aspect of the pathophysiology. For example, because atherosclerosis is a focal disease occurring predominantly in the areas of disturbed flow, marked by low and oscillatory shear stress, it would be desirable to study the areas of naturally-occurring disturbed flow in the animal models. Alternatively, based on our understanding of the vascular mechanobiology, researchers can create areas of disturbed flow using surgical methods to mimic the shear stress profile of the regions of atherosclerosis.
The first paper entitled "Novel Animal Models of Atherosclerosis" by Rezvan et al. provides a brief review of the common animal models used in studying atherosclerosis, with an emphasis on the partial carotid ligation model, which has been used to demonstrate the direct role of disturbed flow in atherosclerosis. Using this animal model, Rezvan et al. discusses the role of disturbed flow characterized by low and oscillatory shear stress on endothelial gene expression, leading to subsequent endothelial dysfunction and atherosclerosis [1]. The second review article entitled "Role for SUMOylation in disturbed flow-induced atherosclerotic plaque formation" by Abe, Le and Heo provides a timely overview of the current state of research in mechanosensitive signal transduction pathway involving SUMOylation that mediates endothelial dysfunction, a key step in atherogenesis, in response to disturbed flow [2]. The third article "Study on the role of microtubules on T cell migration under confined environments" by Park and Doh reports the alterations in morphologies and migratory phenotypes of $\mathrm{T}$ cells by geometrical confinements [3]. Since massive infiltration of $T$ cells occurs in many diseases related with local inflammation such as atherosclerosis, asthma, lupus, and cancers, controlling migration of immune cells can be an effective therapeutic strategy to treat such immune-related diseases.

The fourth article "Lipid bilayer control of nascent adhesion formation" by Son and Butler provides a succinct and timely review of the state of the cell adhesion regulation, focusing on the role of lipid bilayer and its components including cholesterol in nascent adhesion formation [4]. Focal adhesions, made of clusters of integrins, are one of major mechanosensors that transduce extracellular mechanical and chemical signals into multiple regulatory changes of cell behaviors and fate. The last article by Kim et al. highlights the importance of matrix dependent responses of kerationocytes in the context of skin epidermal wound healing [5]. The phenomenon of re-epithelialization of keratinocytes is pivotal to wound healing, and bears resemblance to epithelial-to-mesenchymal transdifferentiation (EMT) during development. Control and regulation of mechanical forces occurring both intrinsically and extrinsically have been shown to be effective in reducing abnormal scarring at the wounds. Thus, understanding this wound-triggered migratory phenotype of keratinocytes could shed novel insights into this process, and may lead to a new therapeutics approach. In addition, the knowledge and mechanobiological principles can be used to design and construct an experimental skin model, which can be used to screen the drugs and cosmetics, partially replacing animal models.

Mechanical stimuli play a critical role in many physiological processes and these mechanical stimuli are closely related to cell proliferation and differentiation, and tissue degeneration 
and development. Thus, in vitro cell culture system must also be designed to mimic realistic physicochemical microenvironment of the cells [6]. Re-evaluation of human pathophysiology in the context of mechanobiology may provide us a unique opportunity to identify novel targets for therapeutic intervention in a variety of diseases. Further, mechanobiological perspectives could offer fresh insights into many diseases for the clinical, bioengineering, and scientific communities.

Finally, we guest editors would like to express our gratitude to the authors who contributed in this special issue in the field of mechanobiology and diseases. We would also like to acknowledge the support from the Managing Editor (Prof. Jae Sung Lee) and Editor-in-Chief (Prof. Sang-Hoon Lee) of the Biomedical Engineering Letters.

\section{REFERENCES}

[1] Rezvan A, Sur S, Jo H. Novel animal models of atherosclerosis. Biomed Eng Lett. 2015; 5(3):181-7.

[2] Abe J, Le NTL, Heo K. Role of SUMOylation in disturbed flowinduced atherosclerotic plaque formation. Biomed Eng Lett. 2015; 5(3):162-71.

[3] Park H, Doh J. Study on the role of microtubules on T cell migration under confined environments. Biomed Eng Lett. 2015; 5(3):188-93.

[4] Son S, Butler P. Lipid bilayer control of nascent adhesion formation. Biomed Eng Lett. 2015; 5(3):172-80.

[5] Kim M, Gweon B, Koh U, Cho Y, Shin DW, Noh M, Shin J. Matrix stiffness induces epithelial mesenchymal transition phenotypes of human epidermal keratinocytes on collagen coated two dimensional cell culture. Biomed Eng Lett. 2015; 5(3):194-202.

[6] Park J, Muller M, Kim J, Seidel H. Fabrication of a cell-adhesive microwell array for 3-dimensional in vitro cell model. Biomed Eng Lett. 2015; 5(2):140-6. 